\title{
AOR
}

Selected Papers of \#AolR2021:

The 22nd Annual Conference of the

Association of Internet Researchers

Virtual Event / 13-16 Oct 2021

\section{TRACKING THE PILLING PIPELINE: LIMITATIONS, CHALLENGES AND A CALL FOR NEW METHODOLOGICAL FRAMEWORKS IN INCEL AND MANOSPHERE RESEARCH}

\author{
Debbie Ging \\ School of Communications, Dublin City University \\ Shane Murphy \\ School of Communications, Dublin City University
}

The manosphere is an online network of disparate and sometimes conflicting, sometimes overlapping assemblages. These formations are united in their antipathy toward feminism, their reliance on evolutionary psychology and their belief that Western civilization is under threat. In recent years, a growing body of scholarship on the manosphere has emerged from a range of disciplinary perspectives. Much of this work sits within internet studies but there are also significant contributions from gender studies, social psychology and terrorism / cybersecurity studies.

The purpose of this paper is to take stock of the current research, to identify methodological limitations, and to propose some new interdependent research frameworks and methods. To date, much of the work conducted on the manosphere and its various subgroups, of which incel is the most frequently researched, relies on gathering a dataset from one platform and subjecting it to either manual or machine analysis to identify key themes or characteristics. This categorisation has been necessary and important, but its frequent replication at this point, we argue, has led to a stagnation of knowledge, and does not facilitate theory building or intervention.

Notwithstanding some excellent theoretical framings from internet studies (Massanari, 2015; Marwick and Lewis, 2017) social psychology (Allan, 2016), gender and media studies (Ging, 2019; Braitch and Banet-Weiser, 2019), sociology (Kimmel, 2018) and terrorism studies (Hoffman et al., 2019; Baele et al., 2019), there remain significant gaps in the research These relate primarily to three defining characteristics of manosphere subcultures: firstly, their anonymity and apparent reluctance to engage with academics, which has resulted in a dearth of interview or survey-based research. Secondly, the dynamic nature of these communities and the speed at which they disband and re-form across disparate platforms means it is difficult to track their spread and development over time. Thirdly, little if anything is known about how users become involved in these

Suggested Citation (APA): Ging, D., Murphy, S. (2021, October). Tracking the Pilling Pipeline: Limitations, Challenges and a Call For New Methodological Frameworks in Incel and Manosphere Research. Paper presented at AolR 2021: The 22nd Annual Conference of the Association of Internet Researchers. Virtual Event: AolR. Retrieved from http://spir.aoir.org. 
communities, i.e. the processes of algorithmic, ideological and psychosocial radicalization or 'pilling' which take place in different internet spaces.

Consequently, we have many useful static snapshots but we are missing the dynamic aspects of how and where ideas travel and interconnect. In addition to this, there is a tendency to think of the various sub-communities as homogenous, despite the significant diversity within them in terms of their agendas, motivations, and levels of involvement. This is especially relevant with regard to how certain vulnerabilities are being exploited in processes of recruitment into incel ideology among socially isolated and neurodivergent men. The dominant perception of incel as continuous with more strategically motivated men's rights formations overlooks the exploitation of genuine - as opposed to performative (betafag) - vulnerability within this subgroup by a range of alt-right and antifeminist actors and influencers. Exploring the heterogeneity within these communities is essential, therefore, to unravelling the dynamics of power that operate within them.

More recently, there is a growing awareness of incel tendencies among several recent American and European far-right attackers, many of whom share a history of hating women, domestic violence and posting misogynistic views online. However, while an abundance of evidence of extremist pipelines exists, it is difficult to access and track. In response to these challenges, we propose new methodological directions that specifically attempt to map dynamic patterns and pathways of anti-women extremism online, based on our recent work on incel formations.

To begin with, we call for a conceptual shift away from thinking of these communities as static, homogenous identity groups operating on isolated platforms, to conceiving of them instead as a multifaceted, ever-evolving online ecosystem, in which activity constantly migrates. We suggest that there is a need to consider a number of pathways, both spatially and temporally: firstly, the spread and extremization of ideas within different components of the incel/manosphere over time; secondly, the migration of ideas across different platforms over time (this relates to flows in both directions, i.e. both mainstreaming and extremization); and thirdly, the spread of ideas to and from other extreme online groups (e.g. alt-right, QAnon, Politigram 'Doomers', etc.), bearing in mind that many of these pathways may also be transnational in nature.

With regard to the first and second pathways, in addition to computational content analysis and in-depth qualitative analysis, we recommend the tracking of activity, such as posting frequency, membership counts and incel/MRA-related keywords. Multi-faceted analysis of this nature, particularly in spaces which were not originally incel, enables us to follow platform adoption dynamics and the various 'pilling pathways' that occur both within and across platforms. In addition to this, it is important to map intensity flows, to determine whether and how ideas become more or less extreme over time.

With regard to radicalisation of and by other extreme communities, particularly the incel to far-right pipeline, we suggest introducing models of the transmission of ideas from research on conspiracy theories. For example, Ward and Voas' (2011) use of the concept of "conspirituality" identifies an important convergence of conspiratorial and new age communities online. Comparing ideological overlaps between communities against the 
timeline of platform adoption also allows us to see whether radicalisation is linked to the adoption of specific platforms.

Finally, interviews, pilling stories and web browser history scroll back analysis (Robards and Lincoln, 2017) can provide vital information regarding pilling pathways. Evans (2018), for example, has analysed redpilling stories shared by fascist activists on white supremacist Discord servers, yielding rich insights into how their platform use changed as they became more 'pilled'. Importantly, they also discuss contemporaneous developments in their real world lives, an important factor which is often left out of discussions of online radicalisation. Approaching the incel/manosphere as a dynamic ecosystem, we argue, will take knowledge of this phenomenon in important new directions, particularly in relation to prevention and intervention, as well as opening up new space for inter-disciplinary collaboration.

\section{References}

Allan, J. A. (2016). Phallic affect, or why men's rights activists have feelings. Men and Masculinities, 19(1), 22-41.

Baele, S. J., Brace, L., \& Coan, T. G. (2019). From "Incel" to "Saint": Analyzing the violent worldview behind the 2018 Toronto attack. Terrorism and Political Violence, 1-25.

Braitch, J. and Banet-Weiser, S. (2019) From Pick-Up Artists to Incels: Con(fidence) Games, Networked Misogyny, and the Failure of Neoliberalism. International Journal of Communication 13.

Evans, R. (2018). From memes to infowars: How 75 fascist activists were 'red-pilled'. Bellingcat, 11 October.

Ging, D. (2019). Alphas, betas, and incels: Theorizing the masculinities of the manosphere. Men and Masculinities, 22(4), 638-657.

Hoffman, B., Ware, J., \& Shapiro, E. (2020). Assessing the threat of incel violence. Studies in Conflict \& Terrorism, 43(7), 565-587.

Kimmel, M. S. (2018). Healing from Hate: How Young Men Get Into--and Out Of--violent Extremism. Berkeley: University of California Press.

Marwick, A., \& Lewis, R. (2017). Media manipulation and disinformation online. New York: Data \& Society Research Institute.

Massanari, A. (2017). \# Gamergate and The Fappening: How Reddit's algorithm, governance, and culture support toxic technocultures. New media \& society, 19(3), 329346.

Robards, B., \& Lincoln, S. (2017). Uncovering longitudinal life narratives: Scrolling back on Facebook. Qualitative Research, 17(6), 715-730. 
Ward, C., \& Voas, D. (2011). The emergence of conspirituality. Journal of Contemporary Religion, 26(1), 103-121. 